\title{
Angst vor dem Coronavirus: Kann ich Patienten ablehnen?
}

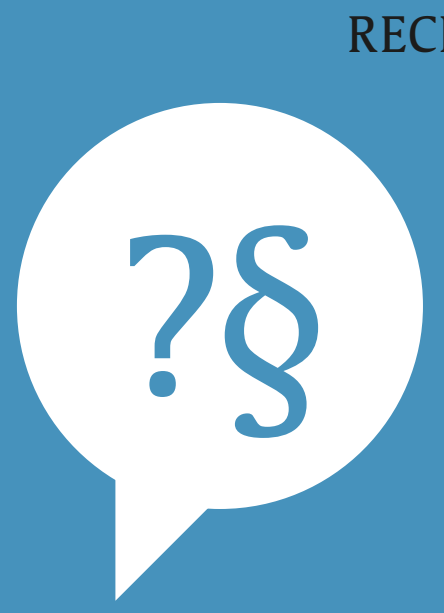

Muss ich einen Patienten behandeln, bei dem aufgrund seiner jüngsten Kontakte ein begründetes Risiko besteht, dass er sich mit dem Coronavirus angesteckt haben könnte? Beispiel aus der Praxis: Ein Patient musste seinen Physiotherapietermin verschieben, um an einer aktuellen Tagung mit Medizinern aus verschiedenen asiatischen Staaten teilnehmen zu können. Nun gibt es Bedenken in der Praxis ...

\section{$\S !$}

EXPERTEN-ANTWORT Als Therapeut mit Kassenzulassung haben Sie einen Behandlungsauftrag gegenüber Patienten mit gültiger Verordnung. Grundsätzlich hat die Behandlungspflicht jedoch da ihre Grenzen, wo eine Behandlung Menschen gefährdet. Die Frage ist, ob der Verdacht einer Infektion mit dem Coronavirus die Behandlungspflicht aushebelt. Wenn man weiß, dass durch einen Patienten die Gefahr einer Infektion für den Therapeuten und für andere Patienten besteht, ist es durchaus vertretbar, diesen Patienten nicht zu behandeln. Denn die Behandlung in dieser Situation kann zu gefährlich sein, bis der Patient den Verdacht hat abklären lassen. Im Zweifelsfalle kann der Patient sich im Krankenhaus behandeln lassen.

Das gilt übrigens für jede ansteckende Krankheit: Wenn sich jemand vorstellt, von dem man weiß, dass er gefährlich für den Therapeuten oder für andere Patienten werden kann, wird man mit der Behandlung warten, bis der Patient wieder insoweit genesen ist, dass nicht mehr von einem Ansteckungsrisiko ausgegangen werden muss und somit keine Gefahr mehr für andere Patienten oder Pra- xismitarbeiter besteht. Als Praxisinhaber sind Sie verpflichtet, Ihre Mitarbeiter zu schützen. Umgekehrt gilt für Mitarbeiter, dass sie nicht in der Praxis arbeiten dürfen, wenn ihnen bekannt ist, dass sie eine ansteckende Erkrankung haben. Das gilt auch beim Verdacht auf Grippe oder sonstige Infektionskrankheiten. Und selbstverständlich sind die gängigen $\mathrm{Hy}$ gienemßnahmen in der Praxis streng einzuhalten. Bitte sensibilisieren Sie dafür entsprechend Ihr Team.

Infektionsschutz in der Praxis ist übrigens nicht erst seit dem Coronavirus wichtig. Auch Patienten mit einer Influenza sind potenziell ansteckend und es gilt, eine Ansteckung zu verhindern. Weisen Sie Ihre Patienten darauf hin, dass die Arbeitskraft Ihrer Therapeuten wertvoll ist, und dass Ihre Praxis daher gezwungen ist, Patienten mit ansteckenden Krankheiten abzulehnen. Sie können auch in Ihrem Behandlungsvertrag oder in Ihren Allgemeinen Geschäftsbedingungen festlegen, dass in Ihrer Praxis Behandlungen bei Patienten mit akuten, ansteckenden Erkrankungen, die eine Gefahr für die behandelnden Therapeuten darstellen, ausgeschlossen sind.

\section{UNSER EXPERTE}

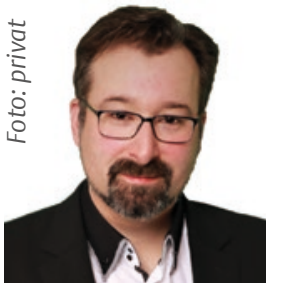

VPT-Bundesjustiziar D. Benjamin Alt ist niedergelassener Rechtsanwalt und berät Sie unter anderem zu Themen wie Arbeitsrecht, Praxisgründung, Praxisverkauf und Praxisübernahme, Haftungsrecht, Abrechnung, Patientenrechte sowie Wettbewerbs- und Werberecht

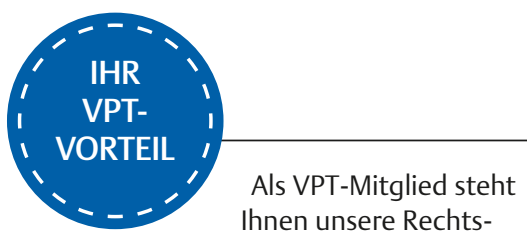

Ihnen unsere Rechtsabteilung zur Seite. Das VPT-MAGAZIN beleuchtet knifflige Fälle aus der Praxis.

Wenden Sie sich mit Ihrer rechtlichen Fragestellung an Ihre Landesgruppe oder an die VPT-Rechtsabteilung, die Mitgliedern eine kostenlose Erstberatung anbietet:

\section{Benjamin Alt}

Eilendorfer Straße 44

52078 Aachen

Telefon: 024195597991

Fax: 024195597992

E-Mail: Alt@VPT.de

Internet: www.RechtsanwaltAlt.de VPT-Sprechzeiten: Montag bis Freitag von 9 bis 14 Uhr. 